\section{ST. GEORGE'S HOSPITAL.}

CONGENITAL FISSURE IN THE HARD PALATE.

Under the care of G. PolLock, Esq.

A RARE form of congenital defect of the palate has been operated on by Mr. Pollock at St. George's Hospital. The patient was a young female. There was a small perforation in the posterior part of the hard palate, situated in the middle line, and divided into two parts by a thin strip of the mucous membrane of the mouth bridging over the fissure. The uvula also was bifid, and the left segment adhered to the edge of the soft palate near the tonsil, producing a very singular appearance. The chief inconvenience which resulted from the defect in the hard palate, was that distinct speech was much interfered with by a sort of whistle produced by the passage of air through the small hole at each utterance. The operation was not very difficult; the edges of the cleft were pared, and the surrounding mucous mem brane was dissected up and united across the cleft; tension being obviated by lateral incisions. Two sutures were then passed through the flaps, and a stout piece of silk through the latera incisions, from one to the other behind the flaps, partly to press the latter together, but also and mainly to prevent the too speedy union of the incisions, which often occurs before union is completed, and produces tension on the wound.

Remarks. It may be observed that Mr. Pollock usually adopts the plan of freeing the mucous membrane from the hard palate by dissection carried on from the lateral incisions towards the cleft. This is not only more easy to do in the majority of cases, but has the important advantage of leaving a perfectly fresh and uninjured edge, when the latter has been pared. If the dissection is carried on from the cleft outwards, it is difficult to avoid bruising the edges which are to be united, and which are thus, of course, rendered more liable to slough.

\section{ST. BARTHOLOMEW'S HOSPITAL。 NECROSIS OF THE FEMUR.}

Under the care of E. Standey, Esq.

[From Notes by E. BARKER, EsQ., House-Surgeon.]

At St. Bartholomew's Hospital, Mr. Stanley operated again upon the case of necrosis of the lower end of the femur, mentioned at page 102. There was still some dead bone in the outer part of the femur, and a sinus existed, from which the probe passed into the shaft and touched dead bone. The present operation consisted in making an incision of four inches in length on the outer side of the limb, and removing part of the outer wall of the bone with a fine saw, when some pieces of necrosed bone were readily found and removed. Two of the pieces measured two inches each in length, and there wer many smaller. The operation was very long and tedious, and the patient was kept under chloroform for an hour and a half but there was no considerable hæmorrhage. A good deal of sickness followed this protracted exhibition of chloroform. The result of this case will be given at a future time.

\section{CITY DISPENSARY.}

\section{CONTRIBUtion to THE PATHOLOGY OF INTRATHORACIC} CANCER.

By John Cockle, A.M., M.D., Physician to the Dispensary ; and to the Margaret Street Dispensary for Consumption, etc.

In a paper, published in the Association Medical Journal for November 3rd, 1854, I endeavoured to point out the occasional difficulty of diagnosis characterising some forms of intrathoracic cancer. The case reported was one of nodulated cancer of the lurigs. The intervening parenchyma being healthy, and the signs of pressure absent, no physical phenomena, during life, revealed the character of the disease. The present contribution illustrates the difficulty of differential diagnosis, even when well marked physical changes lent their aid to the physical investigator.

Mary Firmin, aged 26, of healthy family, was admitted under my care June 28th, 1856.

Previous History. She has felt pain in the lower part of the left side, occasionally, since her marriage, five years ago. There is reason to believe that a blow was received on the side about this period. At Christmas, she began to suffer from shortness of breath. For the last four months, the dyspnca and pain have been greatly increased; the latter extending to the epigastrium, and passing backwards between the shoulders. She is, in addition, troubled with cough, attended with thick yellow expectoration. Blood has never been observed in the sputa. At the commencement of this period, she was compelled to keep her bed, and could lie only upon the right side. At the end of a fortnight, she was enabled to resume her domestic duties. She suffered subsequently several relapses, which confined her to. her bed, and from which she again partially recovered. Shehas so continued to the date of admission.

Present State. The patient does not suffer so much from the pain in her side, but feels her breath very short. She complains of pain in the back of the neck; she coughs a great deal and expectorates yellow phlegm; suffers from palpitation, and feels her heart beating upon the right side; feels sick in the morning; perspires at night, and feels very faint; considers she is decidedly losing flesh. The bowels are regular, and there is diuresis. The catamenia are generally regular, and upon the last occasion were profuse; there is no swelling of the ankles. She can now lie only upon the right side.

Inspection. She is of short stature, somewhat thin, of fair complexion, with light-coloured hair. There is no great action of the nares during respiration. There are observed to be distension of the cervical veins, and pulsation of the right jugular immediately above the clavicle; as well as distension of the mammary veins at the axillary line, more marked upon the left side. The heart pulsates upon the right side between the second and third ribs down to the right nipple. There is marked fulness of the left mammary region, and apparent contraction at the left base anteriorly and laterally. There is a blister mark under the clavicle. The respirations are 38 per minute, exaggerated in type.

Palpation. The cardiac impulse is absent over the normal site, and the normal fremitus is absent over the left side. Pulse 108, small, weak, regular, equal in both wrists.

Percussion. There is marked dulness from below the left clavicle over the left side, anteriorly, laterally, and posteriorly, excepting at the upper and vertebral angle of the scapula. The dulness passes the median line anteriorly towards the right.

Measurement. Three inches below the nipple, the right side. measures $14 \frac{1}{2}$ inches, the left $13 \frac{1}{2}$; above the nipple, the right side measures $15 \frac{1}{2}$ inches, the left 16 .

Auscultation. No respiratory murmur is audible over the left side, with the exception of somewhat hoarse murmur immediately below and above the left clavicle, and at the upper and vertebral angle of the scapula. No ægophony is heard. Upon the right side, laterally and posteriorly, exaggerated blowing respiration is heard. 'I'he maximum of the heart's sounds is over the site of visible impulse; these sounds are normal, and unattended by murmur.

July 4th. There were dyspnœa, and cough with scanty yellow expectoration. The patient lay upon the left side, and had a distressing sensation of the heart beating upon the right side. Last night, the lower part of left side seemed swollen. The kidneys act freely. The patient has pains in the neck, ascending to the face. The pulse, respiration, etc., and physical phenomena, are unchanged.

July 8th. Dyspnœa has increased; the cough is relieved; there is no expectoration. The patient complains of pain in the chest. Dorsal decubitus. Respirations 40 ; pulse 120, small and weak; perspirations.

July 11th. Dyspnœa is increased; the cough is still less; the decubitus is left-sided or dorsal. The kidneys do not act so freely. Friction sound is heard at the inferior part of the left side; the breath-sound is somewhat more distinct at the spine. About this period, the patient suffered severe relapse, with increase of dulness and perfect absence of respiratory murmur in the clavicular and postero-superior region.

Some few weeks subsequently, the patient was admitted into. St. 'Thomas's Hospital, under the care of Dr. Risdon Bennett. This physician thought it advisable, in order to clear the diagnosis, to recommend an exploratory puncture in the ordinary site of empyema. A small quantity of blood alone escaped; shortly afterwards, an oblong diffused tumour appeared in the left axilla; and now Dr. Bennett felt confirmed in the sus picion, that the case was one of malignant growth.

The patient again came under my notice on October 24th. The symptoms were steadily progressive, and the following phenomena were then accurately noted.

Inspection. Emaciation is general; the face is flushed; a large distended vein runs down the forehead. The respirations exaggerated in type, are 32 per minute; pulse, 125 , weak, equal in both radials. The jugular veins are moderately distended 
and pulsating; they are intensely distended during coughing. The left post-clavicular region is somewhat fuller than the right. There is great prominence of the left side, particularly at and above the nipple. The spine is straight; the inferior angle of the left scapula is one inch further from the spine than the right. The left scapula is almost motionless during respiration. There is general fulness of the left side posteriorly. There is permanent distension of the mammary veins, more particularly the left. A diffused flattened tumour exists in left axilla, corresponding to the axillary line: it is painful at times, and always tender upon pressure. A scar of puncture is seen four inches below the axilla, a little to the left of the axillary line, between the fifth and sixth ribs. The abdomen is tumid. There is anasarca of the inferior extremities (increasing).

Palpation. Vocal fremitus is entirely absent over the left side, before and behind. There is well marked sensation of fluctuation between the third and fourth ribs anteriorly, immediately above the nipple. The apex of the heart is felt one inch to the right of the right nipple; the base apparently corresponds to the osteo-cartilaginous junction of the second right rib.

Measurement. In the axilla, the left side measures 16 inches; the right side, 153 inches. Immediately above the nipple, the left side measures 17 inches; the right side, $15 \frac{1}{2}$ inches. Three inches below the nipple, the left side measures $14 \frac{1}{2}$ inches; the right side, $15 \frac{1}{4}$. The left nipple is two inches higher than the right. Both are equidistant from the median line.

Percussion. The left side is universally dull, anteriorly and posteriorly; the dulness passes the median line in front, and extends between one and two inches to the right. The stomachal note is heard in the left hypochondrium. Upon the right side, tolerable resonance exists laterally and posteriorly. The abdomen yields a markedly tympanitic note above, but acquires a comparatively dull character in the lower region, with increased resistance.

Auscultation. Immediately beneath the left clavicle, distant tubular breathing is heard; while, over the remaining portion of the left side, the respiratory murmur is absent. The heart's sounds are heard over the mammary region, diminishing inferiorly. Upon the right side, blowing respiration is heard under the clavicle, with râle sonore during inspiration, extending down the lung, laterally and posteriorly. Posteriorly, the respiratory murmur retains, generally, the blowing character; but rîle at times mingles with the acts of both inspiration and expiration, acquiring with the latter the character of shrill sibilus. Vocal resonance is somewhat exaggerated over the entire right side of the chest. The maximum of the heart's sounds remains as before stated.

Subsequent examinations did not elicit any additional fact of importance, with one exception; viz., during the last three weeks of life, the apparently gradual extension of a weak respiratory murnur over the middle and posterior portion of the left lung.

The infiltration of the lower extremities, and the abdominal effusion, gradually increased, and the function of the kidneys as gradually became impaired; the dyspnœa became, pari passu, more distressing, and attended with suffocating paroxysms, and permanent semicyanotic tint of the nails. So marked was still the sense of fluctuation upon coughing between the third and fourth left ribs, that I was tempted to alvise the introduction of a fine exploring needle over the most prominent portion of the swelling, with the view to momentary relief, should empyema coexist. The operation gave pain, and only a drop or two of blood trickled down the groove of the needle. During the last three days of life, signs of centric pressure developed themselves, and nourishment could only be taken in the erect and forward position. Death, apparently by asthenia, occurred in the latter end of December.

Autopsy. Present: Dr. Sibson, Dr. F. C. Webb, Mr. Selwood, and Mr. Broughton. A general tendency to decomposition was observed. Upon opening the thorax, a large tripartite mass was seen, exceeding in aggregate bulk an adult head, and of so semifluid a consistence that, in detaching it from its connexions, large quantities of grumous blood and encephaloid matter escaped. The portions of the mass were invested with a fine bounding membrane of glistening character. The large rounded portion occupied nearly the entire left side of the chest, crushing the lung along the vertebral column, and adhering to its anterior face. Firm adhesions existed on this side throughout, particularly between the tumour, spine, and mid portions of the fourth, fifth, and sixth ribs, which latter were markedly softened, and formed a medium of 231 connexion between the tumour and the oblong swelling upon the outer chest-wall. The tumour had carried the heart before it as far as the right nipple, but did not itself extend far beyond the right costal cartilages. By this encroachment, however, together with that of the displaced heart, the right lung also was lessened in volume to the extent of at least one-half, and bound down by adhesions. The second portion of the tumour, of the size of an orange, nearly separate from the larger mass, lay behind the manubrium sterni; and a still smaller portion, of the shape of a fig, was situate below the xiphoid cartilage. The heart was normal in size and structure. Considerable effusion existed in the peritoneal cavity. The liver and spleen, etc., were in a physiological condition.

Microscopic examination of the tumour revealed the characteristic elements of malignant growth.

Remarks. The case detailed is replete with practical in terest. In its early stage, there were, as it appears to me, some grounds for regarding it as one of empyema, for which affection, indeed, I mistook it. It may, therefore, be instructive briefly to analyse the symptoms, with the view to determine how far such an opinion was tenable. The early symptoms were pain in the left side, cough with catarrhal expectoration, dyspnœea, difficulty of lying upon the affected side, constitutional excitement, etc. The case was at this time considered and treated by her then medical attendant as one of ordinary pleurisy. At the mid period of the history of the case, both vital symptoms and physical signs appeared to conform to such a view. These were pain, cough, dyspnœa, oppression, and hectic phenomena, as emaciation, night perspirations, small rapid pulse. On inspection, the side was enlarged (markedly so in the mammary region) and motionless during respiration; the percussion sound was dull; respiratory murmur and vocal fremitus were annulled, except beneath the clavicle and near the spine; the heart was displaced; the decubitus upon the affected side.

It is scarcely necessary to recapitulate the phenomena of the later phase of the disease. From the period of the puncture test and the appearance of the external swelling, no rational doubt could be entertained as to the presence of intrathoracic tumour, even assuming the coexistence of empyema to a greater or less extent. In further support, however, of the diagnosis of effusion in the first and second stages of the disease, it may be argued, that left sided pleurisy is by far the most frequent, and that contraction ordinarily commences at the base; so that, remembering the several attacks and partial restorations, it was not unreasonable to infer that such adhesions had commenced below, and acquired sufficient firmness to prevent gravitating effusion from renewed attacks. Such effusion might, therefore, thus occasion bulging of the mid portion of the chest, and even tend to the production of an empyema of necessity. The pericardium also, from its extreme traction to the right, might have dragged the left side preternaturally inwards, and further augmented the apparent contraction of the base. Should it be contended, however, that the marked enlargement of the mammary region, the extent of dulness to the right of the mesial line, the enlargement of the mammary veins, the persistent pain, and frequently recurring attacks, the anasarca, etc., should have awakened snspicion of solid growth, we can only remark, that even these signs do not suffice for a differential diagnosis. Many cases are on record of empyema associated with all these signs to a greater or less extent. (See particularly Gintrac, Diagnosis des Affections Thoraciques Mohr, Monograph des Empyems ; Heyfelder, Essay on Chronic Pleurisy, in his Studien in Gebiete der Heilwissenschaft; Broussais, Phlegmasies Chroniques ; Andral, Clinique, etc., art. iv, obs. 260 ; and particularly that most singular case of relapsing empyema recorded in the London Medical Repository.)

The sensation of fluctuation so evident in this case, and caused by the semi-liquid character of the mass, has been indicated by other observers in malignant intrathoracic disease, especially by Swett, Diseases of the Chest, p. 335 ; and is constantly pointed out clinically by the surgeon in fungoid disease occurring in parts more accessible to observation. It must, therefore, not be lost sight of in the category of phenomena common to internal malignant disease.

One question must be left for the reader to solve, viz., the origin of the disease. Were the signs of pleuritic irritation to be regarded as the result of changes induced by a tumour of independent growth, or was the mass the result of a metamorphose of a primary albuminous pleuritic effusion, as described by Zehetmeyer (Grundzïge, p. 138)? An argument against this view would be the apparent absence of cancerous growth in other organs-a consequence considered as almost 
necessary by this observer. (See, moreover, a most interesting case, detailed in p. 339 of his work.)

There yet remains a phenomenon in the case I have recorded which requires comment; I allude to the partial reappearance of a weak respiratory murmur over a portion of the postero-middle region, towards the close of the case. If this were not a conducted murmur from the right lung (which was doubted by some observers), I can only explain it on the hypothesis that a portion of the tumour had yielded, and permitted part of the left lung to expand into its place.

Finally, with reference to the differential diagnosis of intrathoracic tumour: when we consider the possible sources of error inseparable from the very nature of certain cases of chest affections and their associate signs, such as those very recently described by Drs. Moutard-Martin and Oulmont (Med.-Chir. Review, Oct. 1856); and when we find how, at times, the very ablest observers have been at fault in the diagnosis of malignant disease of the chest, alike from the consideration of the symptoms and the physical signs (the latter, perhaps, are at their minimum of value here), we cite, in special illustration, the well known cases of Boerhaave, Corvisart, Graves, and Stokes, etc., others of less ability may well be excused for hesitancy and doubt. Indeed, it would appear, from a careful collation of the cases extant, that where the family history shows an alsence of cancerous disease, where the expectoration is simply catarrhal, marked signs of centric pressure absent, and no external tumour present, the physical signs and symptoms corresponil so closely with those of pleurisy, either general or circumscribed, that the diagnosis of intrathoracic cancer, at its early stages, is in most cases difficult, and in others, perhaps, occasionally impossible. At the same time, it must be borne in mind that relapsing pleurisy, with pain more or less constant, and radiating far and wide, combined with alteration of the chest-wall, and obstinately intractable to treatment, should at all times excite grave suspicion.

\section{Oritimal Communitations.}

\section{INFLAMMATORY ACTIONS, AND THEIR TREATMENT.}

By C. Handfend Jones, M.M., F.R.S., Assistant-Physician to St. Mary's Hospital.

[Continued from page 216.]

If we regard therapeutic agents as tests of the character of the actions going on in the living body, it may be of much interest to consider some of them in their bearing upon the subject before us. Let us take first the ordinary reputed remedies for sthenic inflammation.

Venesection must be considered as addressed chiefly to the attending constitutional disturbance, rather than to the local affection; it works its end, when beneficial, by taking off the stress of the circulating current, and diminishing the force of the heart's contractions, as indicated by the softer, more yielding, and sometimes slower pulse. It is to be regarded as a heart and artery sedative. 'i'he sedative action upon the arteries is shown by their becoming less contracted, and yielding more to the force of the heart, and to the pressure of the linger in exploring the pulse. Thus a small, wiry pulse will become larger and softer under bloodletting, because the contractility of its coat (muscular) is diminished. The heart will now act more freely and send the blood more equally over the general system, and so the local determination will be relieved. The increased tolerance of bloodletting in sthenic inflammation is a very striking circumstance, and cannot be well referred, I think, to any other cause than to an increase of nervous force in those ganglia or centres which influence the heart's action. A strong contractile action of the heart, and a tense, contracted state of the arterial coats generally, must imply, I think, increased innervation of these parts, since the opposite state is observed in all morbid conditions wherein nervous power is materially lowered. At the present time, together with an absence of all conditions that require or justify venesection (speaking generally), I meet with instances of impaired action of the heart, sometimes amounting to serious danger. This is associated with other symptoms of depressed nervous power, which appear to be most reasonably attributed to some kind of epidemic influence, much resembling that of malarious disease. The reaction which occurs after venesection, and which is stilled by opium, can hardly be otherwise regarded than as a phenomenon produced through the nervous system. That venesection (when performed so as to abstract blood rapidly) can produce also local effects, is shown by the occurrence of nausea and vomiting, and the breaking out of perspiration, which it not unfrequently occasions. In this case it acts evidently in the way of shock through the nervous system, as the same phenomena may result from the sudden rupture of a bloodvessel within the cranium.

That local bleedings exercise a sedative effect upon an irritated tissue in sthenic inflammation seems to be certain, not only from the good effects so commonly observed from it, when performed on a part whence blood can be directly abstracted from loaded vessels, but much more from the benefit obtained when this is not possible. No one can doubt that leeches applied to the epigastrium will very materially relieve an inflamed stomach, yet how is it conceivable that this relief is effected? It surely is not by the quantity of blood abstracted from the general system (as in venesection), and it certainly is not by any direct depletion of the gastric vessels themselves. We seem to be absolutely shut up to the conclusion, that the modi fication of one part in its nutrition can affect an adjacent, in such wise that a state of irritation, and active determination of blood thither, shall be diminished. The same remark applies to the leeching of the temples to relieve hypermic and irritated conditions of the encephalon, and to many other like cases. Had it not been for experience, we should have thought the proceeding quite irrational; but in this, as in other instances, we are instinctively, if not divinely, led to do the right thing first, and at a later period we come to reflect, and try to discover the principle, or reason, of our practice.

Cold, it will not be questioned, exerts a sedative action on an irritated tissue. It benumbs nerves, and impairs the play of muscles, if at all considerable, and it is, therefore, likely to act similarly on an inflamed part. In orchitis, Mr. Curling has proved its adequacy to cure by repeated and prolonged applications; and in meningitis and maniacal excitement, its beneficial action is well known. Similarly, in inflammatory states of the stomach, lumps of ice are administered with much advantage; but, if the vomiting depend merely on deranged innervation, they are productive of little or no good. In all these cases, it seems impossible to refer the good efrects of the cold solely to its causing contraction and shrinking of the bloodvessels, though this operation may have some share in the result. In fact, the sedative power of coll is most strikingly shown by its nower of arresting the circulation in a part, and causing it to become blue, or livid, from stagnating or slowly moving blood. This is unlike the usual action of cold in rendering a part pale and bloodless by contracting its arteries; the retardation of the blood must depend on a paralysis of the normal nutritive processes, which have so much intluence in modifying its distribution.

Antimony and ipecacuan have strong claims to be regarded as sedatives of certain tissues, although their local application in a more concentrated form is strongly irritant. 'That they will subdue sthenic pulmonary inflammations is for me a certain fact, as also that they will agrravate and increase asthenic. I suppose also that their power, when given early as emetics, to arrest or check acute tonsillitis, orchitis, and adenitis, may reasonably be admitted. The benefit produced by ipecacuan in sthenic dysentery, when given in frequently repeated closes, is also, I believe, unquestionable. The powerful effect of antimony for good in croup is not a matter of doubt. Whatever be the exact way in which these remedies act, whether directly on the tissue, or through the nerves, it must be, I think, admitted that they do modify that morbid tendency to accumulation and arrest of blood in the suffering parts, which we have regarded as a main feature of sthenic inflammation. They are contrastimulants. The muscular relaxation and general debility induced by full doses of antimony and ipecacuan, as well as their diaphoretic action, show that they must act powerfully on contractile tissue, voluntary and involuntary, and this in a way of paralysing and weakening its power. This points also in the direction just indicated.

Colchicum, with its magical action in reducing the articular inflammation of sthenic gout, must also be alluded to. Its action in acute rheumatism, though much more uncertain, is also, according to Dr. Watson's testimony and Dr. Fuller's, almost as striking. My experience agrees with that of these physicians, as to its being most appropriate to the synovial or articular form of rheumatism. These instances of the beneficial operation of colchicum are not necessarily conncted with its 